\title{
Brain MRI, lumbar CSF monoamine concentrations, and clinical descriptors of patients with spinocerebellar ataxia mutations
}

\author{
J J Higgins, J D Harvey-White, L E Nee, M J Colli, T A Grossi, I J Kopin
}

\begin{abstract}
Objectives-To serially assess changes in lumbar CSF biogenic amines, radiographic characteristics, and neurological signs in 34 patients with dominantly inherited ataxia.

Methods-Mutational analysis was used to identify genetic subgroups. Annual assessment of lumbar CSF monoamine metabolites using a gas chromatographic/mass spectrometric method and morphometric measurements of the cerebellum, pons, and the cervical spinal cord on MRI were analysed for each patient and compared with normal controls.

Results-Patients with CAG trinucleotide repeat expansions on chromosome 6p (mutSCA1) and chromosome 14q (mutSCA3) had only about one half the normal concentrations of lumbar CSF homovanillic acid (HVA) whereas, 5hydroxyindoleacetic acid (5-HIAA) concentrations were similar to those in age matched normal subjects. The HVA and 5-HIAA concentrations in clinically similar patients without mutSCA1 or mutSCA3 were normal. One year after the first study, HVA concentrations were reduced by a mean of $22 \%$ regardless of the patient's SCA mutation. Abnormalities on MRI were consistent with a spinopontine atrophy in patients with mutSCA3, spinopontocerebellar atrophy in patients with mutSCA1, and "pure" cerebellar atrophy in patients without these mutations.

Conclusions-Quantitative MRI measurements were not useful in monitoring progression of disease but lumbar CSF HVA concentrations and total scores on a revised version of the ataxia clinical rating scale seemed to progress in parallel.
\end{abstract}

$(\mathcal{F}$ Neurol Neurosurg Psychiatry 1996;61:591-595)

Keywords: spinocerebellar degeneration; cerebellar ataxia; biogenic monoamines; nuclear magnetic resonance

Six genotypes have been assigned to a group of neurodegenerative disorders called the autosomal dominant ataxias. ${ }^{1-9}$ Two of these disorders are characterised by an expanded CAG trinucleotide repeat at the SCA1 locus on chromosome $6 \mathrm{p} 1$ (mutSCA1) and at the SCA3 locus on chromosome 14q
(mutSCA3). ${ }^{15910}$ Few studies have evaluated lumbar CSF monoamine metabolites or quantitative MRI abnormalities in groups of patients with dominantly inherited ataxia. ${ }^{11-16}$ Thus the results of clinical studies performed before the discovery of mutSCAl and mutSCA3 are difficult to interpret. Patients with dominantly inherited ataxia and "pure" cerebellar cortical atrophy on MRI have low lumbar CSF concentrations of 5-hydroxyindoleacetic acid (5-HIAA) and homovanillic acid (HVA). ${ }^{16}$ By contrast, necropsy specimens from patients with mutSCA1 show low concentrations of striatal HVA with normal to increased striatal concentrations of 5-HIAA. ${ }^{1718}$ Before products containing Ltryptophan were associated with the eosinophilia-myalgia syndrome, ${ }^{19}$ the serotonin precursor, L-5-hydroxytrytophan (5-L-HTP) was found to be an ineffective treatment for patients with dominantly inherited ataxia (Schut-Haymaker type and Machado-Joseph disease). As expected from the lumbar CSF monoamine profile, L-5-HTP did improve static ataxia symptoms in patients with an MRI diagnosis of "pure" cerebellar cortical atrophy and a clinical diagnosis of Harding ADCA type III. ${ }^{1620}$ However, despite a depletion of striatal dopamine and lumbar CSF HVA, levodopa treatment did not ameliorate ataxic symptoms in patients with dominantly inherited ataxia. $^{21}{ }^{23}$ The distinct lack of parkinsonian signs in many patients with mutSCA1 and mutSCA3 implies that dopamine deficiency is not great but suggests that a dopaminergic deficit does play a part in the pathogenesis of these genetic disorders. Even though the gross postmortem neuropathology correlates well with the radiological features of patients with ataxia, ${ }^{1415}$ evaluating patients in treatment groups categorised by MRI findings is unsatisfactory as it ignores genetic heterogeneity. To clarify the topography of MRI lesions and to define the monamine abnormalities in patients with SCA mutations, we longitudinally quantified atrophic changes on MRI, measured lumbar CSF monoamine metabolites, and objectively assessed neurological examinations on a revised version of the ataxia clinical rating scale (ACRS-rev). ${ }^{24-26}$

\section{Materials and methods}

PATIENT SELECTION AND CLINICAL TESTING Informed consent was obtained from each patient before all genetic, radiological, and clinical tests. This study was approved by the 
Figure 1 Representative T1 weighted MRI images $(T R=400 \mathrm{~ms}, T E=$ $16 \mathrm{~ms}$ ) displayed on the image analyser monitor showing the boundaries used to calculate $(A)$ the mediosagittal areas of the cerebellum, pons and posterior fossa and $(B)$ the axial area of the cervical spinal cord.
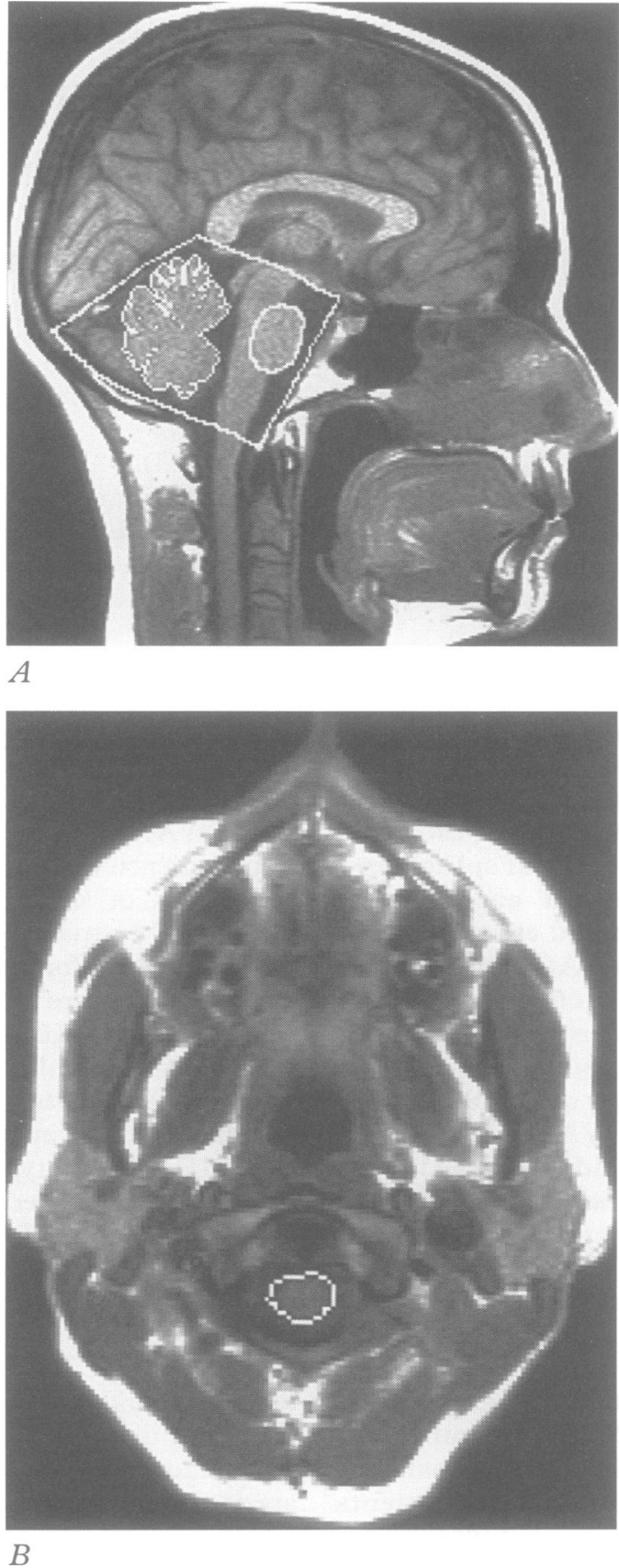

Institutional Review Board of the National Institute of Neurological Disorders and Stroke. Investigators were blinded from the genetic profiles of asymptomatic subjects. Patients from families with ataxia for three or more consecutive generations were recruited by the Neurogenetics Unit. JJH performed all neurological examinations and was blinded to the ACRS scores when the patients were reevaluated at yearly intervals. The ACRS was revised to account for the progressive loss of tone and reflexes in our population of patients by assigning positive scores for decreases in these items (ACRS-rev).

ASSAY OF CSF HVA AND 5-HIAA

After five days on a standard low monoamine diet and after eight hours of bed rest, a lumbar puncture was performed in the lateral decubitus position. Routine CSF studies were per- formed on the initial $4 \mathrm{ml} \mathrm{CSF}$; for assay of HVA and 5-HIAA an additional $10 \mathrm{ml}$ was collected in four $2.5 \mathrm{ml}$ aliquots. All aliquots of CSF were frozen immediately on dry ice and stored at $-70^{\circ} \mathrm{C}$. To minimise the effects of CSF monoamine concentration gradients, the fourth aliquot of CSF was used for analysis. Extraction, derivatisation, and measurement of HVA and 5-HIAA were performed as previously described ${ }^{27}$ on a Hewlett Packard MSD 5970 mass spectrometer/gas chromatograph 5890 with preserved acid extracts of CSF supernatant liquid. The investigators (JDH-W, IJK) analysing the CSF samples were blinded to the patient's clinical and genetic information.

GENETIC TESTING

A pair of fluorescein labelled oligonucleotides flanking the genetic region of interest were used in the polymerase chain reaction to amplify the CAG triplet repeats on chromosomes $6 \mathrm{p}$ and $14 \mathrm{q}$ as previously described. ${ }^{9}$

\section{MRI MORPHOLOGICAL MEASUREMENTS}

Brain MRI was performed twice at a one year interval, using a 0.5 Tesla unit scanner (Picker International Inc, model HPQ). Three $5.0 \mathrm{~mm}$ mediosagittal images were obtained parallel to the longitudinal fissure. The axial area of the cervical spinal cord (CSC; perpendicular to the first cervical vertebra) and the mediosagittal areas of the pons (P; an elliptical pontine area bounded by the anterior surface of the pons, the interpeduncular fossa, and the putative medial lemniscus), cerebellum (Cb) and the posterior fossa ( $\mathrm{PF}$; a quadrangular area bounded by the tentorium cerebelli, the inner table of the skull, and the clivus) were quantified using ANALYZE ${ }^{\mathrm{TM}}$ version 6.2 software (Biomedical Imaging Resources, Mayo Foundation, Rochester, $\mathrm{MN}$ ) on a DIGITAL $^{\mathrm{TM}}$ DEC station 5000/125 (fig 1). Two investigators (MJC, TAG) were blinded to the ACRS scores and the genetic testing results at the time of the MRI analysis. Triplicate measurements of each neuroanatomical area were used to calculate the mean area in pixels. The size of the $\mathrm{Cb}$ and $\mathrm{P}$ were expressed as a ratio to the PF to adjust for variations in head size. Identical areas from 10 normal volunteers were used for comparisons with study participants.

\section{STATISTICS}

StatView ${ }^{\mathrm{TM}}$ version 4.01 software (Abacus Concepts, Inc, Berkeley, CA, USA) was used to compute all statistics. Differences between groups were examined by the Wilcoxon signed rank test. ${ }^{28} \mathrm{~A}$ simple regression model using measures obtained at the beginning of the study as predictor variables and the measurements of these variables one year later as the outcome variables was applied to the data. Residual values were plotted against the predicted values to verify the accuracy of the regression equations. The Bonferonni method was applied to adjust the $\alpha$ level according to the number of comparisons that were tested. ${ }^{29}$ 


\section{Results}

SERIAL CLINICAL FINDINGS

There were two families with mutSCA1, five with mutSCA3, and three without mutSCA1 or mutSCA3 included in the study. Four of six members from one family and two of three members from another family without mutSCA1 or mutSCA3 had the clinical features of Harding ADCA type III. However, the four remaining members from these families without mutSCA1 or mutSCA3 had the neurological signs (supranuclear gaze palsy, dementia, and extrapyramidal signs) associated with Harding ADCA type I. All the families, regardless of genotype, exhibited the phenotypic variability seen in Harding ADCA type I. Neurological findings on the ACRS were similar in the 34 patients (mutSCA1, $\mathrm{n}=7$; mutSCA3, $\mathrm{n}=17$; without mutSCA1 or mutSCA3, $\mathbf{n}=10$ ) examined. All patients had gait ataxia varying from mild to severe. Cranial nerve findings included dysarthria, nystagmus, internuclear ophthalmoplegia, supranuclear gaze palsies, slowed saccadic movements, tongue fasciculations, and coughing dyspnoea. Retinoschisis and Parkinson's syndrome were present in one family without mutSCA1 or mutSCA3 but these features were not found in any of the patients involved in the MRI or CSF analyses. Deep tendon reflexes and muscle tone ranged between extremes. Sensation in the limbs varied from normal to complete loss of position and pain sensation. Limb atrophy, myoclonus, and scoliosis also varied. There were no significant differences in sex, disease duration, age at onset of disease, or total ACRS-rev scores between patients with mutSCA1, mutSCA3, or those patients without these mutations. At the onset of the study, patients without mutSCA1 or mutSCA3 were older $(63.6$ (3.3), mean (SEM)) than patients with
mutSCA1 (35.8 (2.3)) and mutSCA3 (47.1 $(3 \cdot 0))$. In patients that were examined at a yearly interval $(n=14)$, the initial total score on the ACRS-rev was 27 (5) and one year later it was significantly $(P=0.001)$ worse at 45 (7). In patients with mutSCA3 $(n=11)$, the initial total score was 34 (8) and was also significantly $(P=0.02)$ worse one year later at 55 (10). As predicted by a simple regression model, the initial total ACRS-rev scores changed 14 to 16 points in one year in all patients regardless of genotype (fig $2 \mathrm{~A}$ ).

\section{MONOAMINE METABOLITES}

The opening pressure, protein, glucose, and the microscopical appearance of the CSF was normal in all subjects. Lumbar CSF HVA concentrations were about $54 \%$ to $55 \%$ lower in patients with mutSCAl $(\mathrm{n}=7)$ and mutSCA3 ( $n=15$ ), but 5-HIAA concentrations were not significantly different from those of normal subjects $(n=20)$. The ratio of HVA to 5-HIAA was significantly $(P<0.01)$ lower in patients with mutSCA3 than in controls suggesting a monoaminergic neurotransmitter imbalance (table). A second lumbar puncture was performed on nine patients one year after the initial one. In these patients, lumbar CSF HVA concentrations decreased significantly $(P<0.01)$ by 22 (range 0-42)\%. Regression analysis predicted a relation between the initial lumbar CSF HVA concentrations and concentrations one year later (fig 2B).

\section{FINDINGS ON MRI}

Two independent blinded investigators quantified the areas of the $\mathrm{Cb} \mathrm{P}, \mathrm{PF}$, and CSC on $1176 \mathrm{MRI}$ images. In patients without mutSCA1 or mutSCA3 ( $\mathrm{n}=7$ ), quantitative MRI findings were consistent with a cerebellar atrophy $\quad(\mathrm{Cb} / \mathrm{PF}=65.3 \quad(4 \cdot 6) ; \%$ normal
Figure 2 Longitudinal measurements of clinical and biochemical variables in patients with autosomal dominant ataxia. (A) Relation of initial total $A C R S$-rev scores to scores after a one year period of observation. (B) Relation of initial lumbar CSF HVA concentrations to concentrations after a one year period of observation.

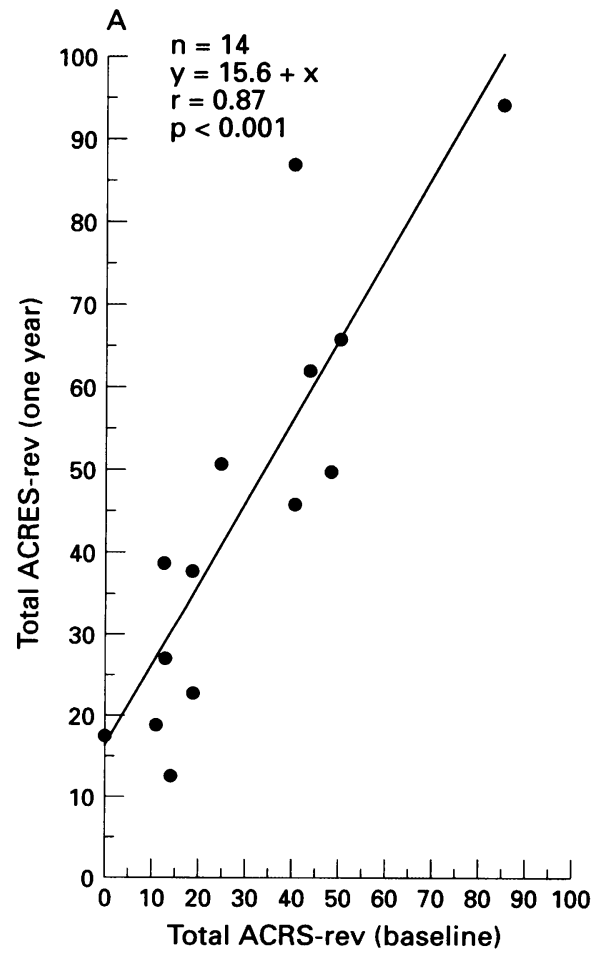

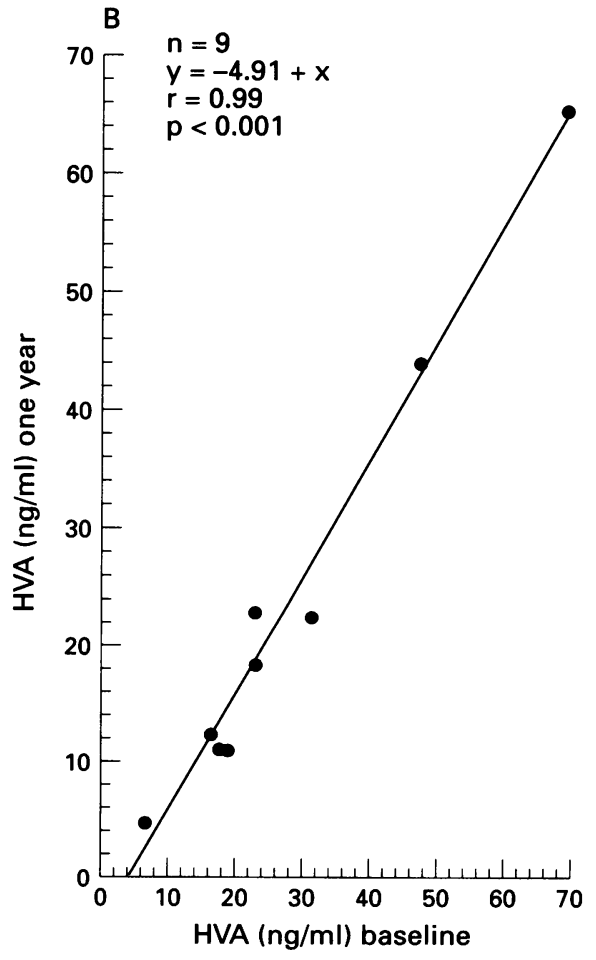


Lumbar CSF monoamine metabolites in normal subjects and in patients with SCA mutations

\begin{tabular}{|c|c|c|c|}
\hline $\begin{array}{l}\text { Genotype } \\
(n)\end{array}$ & $\begin{array}{l}H V A \\
(n g / m l)\end{array}$ & $\begin{array}{l}\text { 5-HIAA } \\
(\mathrm{ng} / \mathrm{ml})\end{array}$ & $\begin{array}{l}\text { Ratio } H V A / \\
\text { 5-HIAA }\end{array}$ \\
\hline $\begin{array}{l}\text { mutSCA1 (7) } \\
\text { mutSCA3 (15) } \\
\text { Othert (8) }\end{array}$ & $\begin{array}{l}18 \cdot 10(2 \cdot 69)(10 \cdot 91-31 \cdot 46)^{\star} \\
17 \cdot 71(2 \cdot 45)(4 \cdot 62-44 \cdot 00)^{\star \star} \\
41 \cdot 22(7 \cdot 81)(8 \cdot 68-68 \cdot 67)\end{array}$ & $\begin{array}{l}11 \cdot 67(2 \cdot 00)(5 \cdot 93-22 \cdot 52) \\
13 \cdot 82(1 \cdot 14)(5 \cdot 44-21 \cdot 66) \\
18.26(3 \cdot 27)(5 \cdot 34-32 \cdot 29)\end{array}$ & $\begin{array}{l}1 \cdot 61(0 \cdot 35)(1 \cdot 09-2 \cdot 02) \\
1 \cdot 38(0 \cdot 18)(0 \cdot 28-2 \cdot 94)^{\star \star} \\
2 \cdot 18(0 \cdot 21)(1 \cdot 10-2 \cdot 85)\end{array}$ \\
\hline Total $(30)$ & $24 \cdot 06(3 \cdot 07)(4 \cdot 62-68 \cdot 67)$ & $14 \cdot 51(1 \cdot 18)(5 \cdot 34-32 \cdot 29)$ & $1.65(0.12)(0.28-2.94)^{\star}$ \\
\hline Normal (20) & $39.08(3.02)(17.03-61.50)$ & $17 \cdot 26(1 \cdot 26)(6 \cdot 67-26 \cdot 40)$ & $2.30(0.12)(1.50-3.39)$ \\
\hline
\end{tabular}

Values are mean (SEM) (range).

.01; Significantly lower compared with normal using the Wilcoxon signed rank test.

subjects without mutSCA1 or mutSCA3. mutSCA = Spinocerebellar ataxia gene mutations; HVA = homovanillic acid; 5-HIAA = 5-hydroxyindoleacetic acid.

(SEM); P $<0.01)$ ) with sparing of the pons $(\mathrm{P} / \mathrm{PF}=100 \cdot 0(6 \cdot 3))$ and cervical spinal cord $(\mathrm{CSC}=86 \cdot 5 \quad(5 \cdot 6)) . \quad$ In patients with mutSCA3 $(\mathrm{n}=19)$ all three neuroanatomical areas seemed to be atrophic $(\mathrm{Cb} / \mathrm{PF}=83 \cdot 1$ $(1.6), \mathrm{P}<0.01 ; \mathrm{P} / \mathrm{PF}=77.0(3.6), \mathrm{P}=0.03$; $\mathrm{CSC}=80(5 \cdot 0))$. Analysis of these same neuroanatomical areas showed severe atrophy

Figure 3 Quantitative MRI findings in patients with autosomal dominant ataxia. (A) The cerebellum/posterior fossa ratio indicates the most atrophy in patients without mutSCA1 or mutSCA3 followed by those with mutSCA1, mutSCA3, and patients at risk for mutSCA3. (B) Pons/posterior fossa ratio and $(C)$ the area of the cervical spinal cord indicate that these regions are relatively spared in patients without mutSCA1 or mutSCA3 and in patients at risk for mutSCA3. Data are averages of triplicate determinations (SEM) from two investigators.
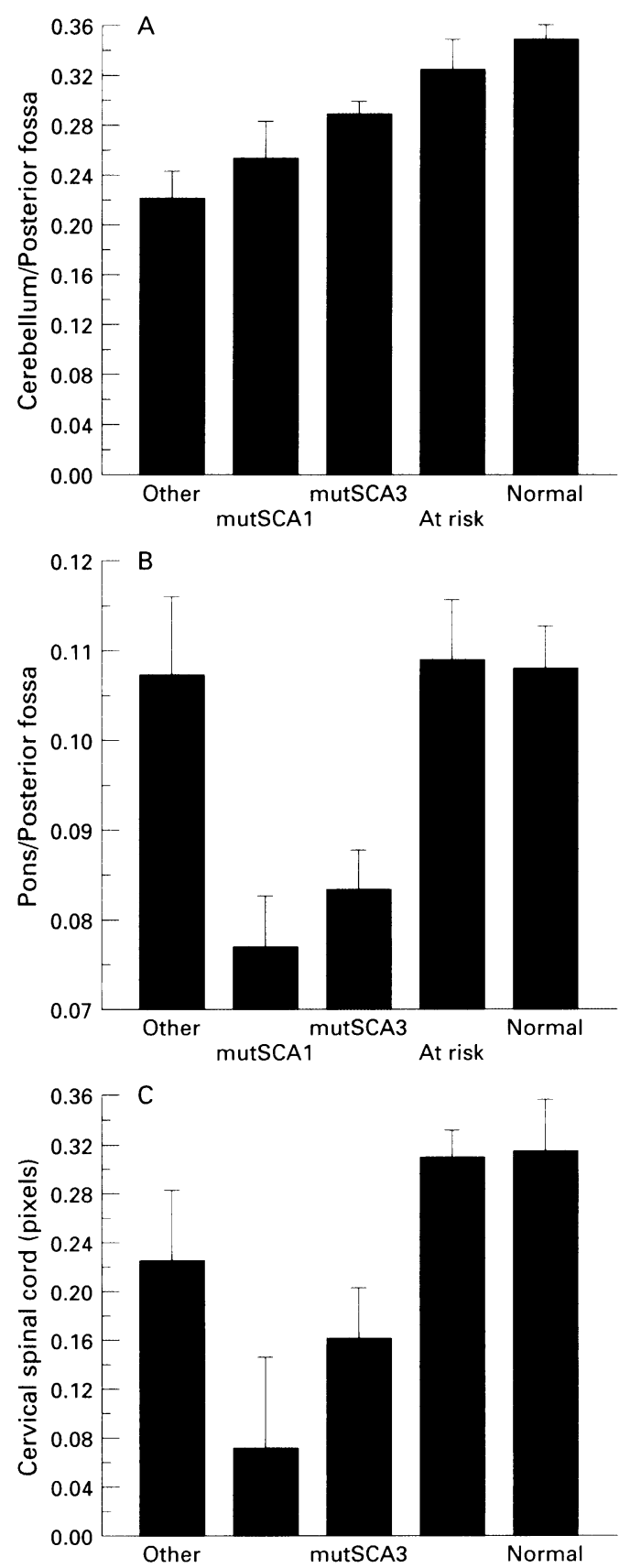

$(\mathrm{Cb} / \mathrm{PF}=72.8(7 \cdot 1), \mathrm{P}<0.05 ; \mathrm{P} / \mathrm{PF}=71 \cdot 4$ $(4.7), \quad \mathrm{P}<0.05 ; \quad \mathrm{CSC}=71.0 \quad(7 \cdot 1)$, $\mathrm{P}<0.05)$ ) in patients with mutSCA1 $(\mathbf{n}=7)$. A CAG trinucleotide repeat expansion on chromosome $14 \mathrm{q}$ was identified in two of the six members at risk. Quantitative MRI testing detected cerebellar atrophy $(\mathrm{Cb} / \mathrm{PF}=75 \cdot 2)$ and cerebellopontine atrophy $(\mathrm{Cb} / \mathrm{PF}=75 \cdot 6$; $\mathrm{P} / \mathrm{PF}=78 \cdot 0)$ in two of these asymptomatic subjects but a correlation with their genetic information could not be made due to the blinded study design (fig 3A-C) There were no significant changes in the sizes of the $\mathrm{Cb}, \mathrm{P}$, or CSC on MRI in any of the study participants over a one year period of observation.

\section{Discussion}

The postmortem lesions in the dominantly inherited ataxias show variable involvement of the substantia nigra, inferior olives, pontine nuclei, Purkinje cells, dentate nuclei, spinocerebellar tracts, anterior horn cells, and peripheral nerves. Presumably, the neuropathological and biochemical lesions seen in patients with mutSCA 1 and mutSCA 3 are not directly caused by their widely expressed gene products. Based on our MRI analysis, it seems that atrophy mainly occurs in the cerebellum in patients without mutSCA1 or mutSCA3. The cerebellum, pons, and spinal cord are all affected in patients with mutSCA1, and a spinopontine atrophy predominates in patients with mutSCA3. These MRI findings are in agreement with the neuropathological findings in patients with mutSCA1 and mutSCA3. ${ }^{30} 32$ The low concentrations of CSF HVA in patients with mutSCA 1 and mutSCA3 correlate with the MRI findings, suggesting a more severe biochemical lesion affecting brainstem dopaminergic pathways. The slightly lower CSF concentrations of 5-HIAA in patients with mutSCA 1 and mutSCA3 may also reflect a diminished contribution from the spinal cord and the cerebellar serotonergic pathways. By contrast, in patients without mutSCA1 or mutSCA3 there does not seem to be a monoaminergic imbalance despite a "pure" cerebellar atrophy on MRI. Other investigators have documented reduced striatal enzyme concentrations of aromatic L-amino acid decarboxylase and tyrosine hydroxylase in patients with severe dopamine loss and a reduction in tyrosine hydroxylase but not aromatic L-amino acid decarboxylase in patients with moderate dopamine loss. ${ }^{17}$ These findings and our current data suggest that serotonergic 
abnormalities in patients with mutSCA1 and mutSCA3 may reflect a functional variation of the serotonergic system under abnormal dopaminergic control. We suggest that this is the reason why patients with dominantly inherited ataxia do not respond to agents which alter serotonin metabolism.

To our knowledge, this is the first report describing serial measurements of biochemical, clinical, and radiological variables in patients with dominantly inherited ataxia. We have found by MRI that the degree of atrophy of the cerebellum, pons, and cervical spinal cord do not change over a one year period. It seems that lumbar CSF HVA concentrations and the total ACRS-rev scores could serve as meaningful biological markers to monitor progression of disease during therapeutic trials. Until the underlying pathogenetic mechanism of the CAG trinucleotide repeat expansion is unravelled, symptomatic treatment is currently the only option. Our data support the notion that combined treatment with serotonergic and dopaminergic agonists may improve the monoaminergic imbalance present in patients with mutSCA1 or mutSCA3. Interestingly, the MRI findings in those at risk for mutSCA3 indicates that the cerebellum may be the first neuroanatomical structure involved in the disease process. However, the relative paucity of abnormal histological findings in the cerebellum of patients with the mutSCA3 implies that the cerebellar afferents originating in the brainstem are the first areas targeted by the disease. ${ }^{933}$ The distinct topography of radiological and biochemical lesions in patients with various SCA mutations also suggests that there may be developmentally regulated injuries to the metencephalon by different genes, gene products, or modifier genes.

1 Orr HT, Chung M, Banfi S, et al. Expansion of an unstable trinucleotide CAG repeat in spinocerebellar ataxia type 1. Nature Genet 1993;4:221-6.

2 Gispert S, Twells R, Orozco G, et al. Chromosomal assignment of the second locus for autosomal dominant cerebellar ataxia (SCA2) to chromosome 12q23-24.1. Nature Genet 1993;4:295-9.

3 Ranum LPW, Schut LJ, Lundgren JK, Orr HT, Livingston DM. Spinocerebellar ataxia type 5 in a family descended from the grandparents of President Lincoln maps to chromosome 11. Nature Genet 1994;8:280-4

4 Gardner K, Alderson K, Galster B, Kaplan C, Leppert M, Ptacek L. Autosomal dominant spinocerebellar ataxia: clinical description of a distinct hereditary ataxia and genetic localization to chromosome 16 (SCA4) in a Utah kindred [abstract]. Neurology 199444 (suppl 2):361.

5 Kawaguchi $Y$, Okamoto $T$, Taniwaki $M$, et al. CAG expansions in a novel gene for Machado-Joseph disease at chromosome 14q32·1. Nature Genet 1994;8:221-8.

6 Stevanin G, Le Guern E, Ravisé N, et al. A third locus for autosomal dominant cerebellar ataxia type 1 maps to chromosome 14q24.3-qter: evidence for the existence of a fourth locus. Am $₹$ Hum Genet 1994;54:11-20.

7 Benomar A, Krols L, Stevanin G, et al. The gene for autosomal dominant cerebellar ataxia with pigmentary macular dystrophy maps to chromosome 3p12-p21.1. Nature Genet 1995;10:84-8.

8 Gouw LG, Kaplan CD, Haines JH, et al. Retinal degeneration characterizes a spinocerebellar ataxia mapping to tion characterizes a spinocerebellar ataxia map
chromosome 3p. Nature Genet 1995;10:89-93.

9 Higgins J, Nee LE, Vasconcelos O, et al. Mutations in American families with spinocerebellar ataxia type 3 :
SCA3 is allelic to Machado-Joseph disease. Neurology 1996;46:208-13.

10 Matilla T, McCall A, Subramony SH, Zoghbi HY Molecular and clinical correlations in spinocerebellar ataxia type 3 and Machado-Joseph disease. Ann Neurol 1995;38:68-72.

11 Rosenberg RN, Nyhan WL, Bay C, Shore P. Autosomal dominant striatonigral degeneration. A clinical pathologic and biochemical study of a new genetic disorder. Neurology 1976;26:703-14.

12 Higgins JJ, Harvey-White JD, Kopin IJ. Low lumbar CSF concentrations of homovanillic acid in the autosomal concentrations of homovanillic acid in the autosomal 1995;8:760.

13 Orozco G, Estrada R, Perry TL, et al. Dominantly inherited olivopontocerebellar atrophy from eastern Cuba Clinical, neuropathological and biochemical findings. $f$ Neurol Sci 1989;93:37-50.

14 Bonni A, del Carpio-O'Donovan R, Robitaille Y, Andermann E, Andermann F, Arnold DA. Magnetic resonance imaging in the diagnosis of dominantly inherited cerebello-olivary atrophy: a clinicopathologic study. Can Assoc Radiol f 1993;44:194-8.

15 Nabatame H, Fukuyama H, Akiguchi I, Kameyama M, Nishimura K, Nakano Y. Spinocerebellar degeneration: qualitative and quantitative MR analysis of atrophy. $\mathcal{F}$ Comput Assist Tomogr 1988;12:298-303.

16 Trouillas P, Renaud CB, Eynard N, Adeleine P. The serotonergic hypothesis of cerebellar ataxia and its pharmacologic consequences. In: Trouillas $P$, Fuxe $K$, eds. Serotonin, the cerebellum, and ataxia. New York: Raven Press, 1993:323-34.

17 Zhong X-H, Haycock JW, Shannak K, et al. Striatal dihydroxyphenylalanine decarboxylase and tyrosine hydroxylase protein in idiopathic Parkinson's disease and dominantly inherited olivopontocerebellar atrophy. Mov Disord 1995;10:10-17.

18 Kish SJ, Robitaille Y, El-Awar M, et al. Striatal monoamine transmitters and metabolites in dominantly inherited olivopontocerebellar atrophy. Neurology 1992;42:1573-7.

19 Hertamann PA, Blevins WI, Mayer J, Greenfield B, Ting M, Gleich GJ. Association of the eosinophilia-myalgia syndrome with the ingestion of tryptophan. $N$ Engl $f \mathrm{Med}$ 1990;322:869-73.

20 Harding AE. The clinical features and classification of the late autosomal dominant cerebellar ataxias: a study of 11 families, including descendants of the "Drew family of Walworth". Brain 1982;105:1-28.

21 Woods BT, Schaumburg HH. Nigro-spino-dentatal degeneration with nuclear ophthalmoplegia: a unique and par tially treatable clinico-pathological entity. $\mathcal{f}$ Neurol $S c i$ 1972;17:149-66.

22 Klawans HL, Zeitlin E. L-dopa in parkinsonism associated with cerebellar dysfunction (probable olivopontocerebellar degeneration). $尹$ Neurol Neurosurg Psychiatry 1971:34: 14-19.

23 Goetz CG, Tanner CM, Klawans HL. The pharmacology of olivopontocerebellar atrophy. In: Duvoisin RC, of olivopontocerebellar atrophy. In: Duvoisin RC, York: Raven Press, 1984:143-8.

24 Pourcher E, Barbeau A. Field testing of an ataxia scoring and staging system. Can $\mathcal{F}$ Neurol Sci 1980;7:339-44.

25 Campanella G, Filla A, DeFalco F, Mansi D, Durivage A Barbeau A. Friedreich's ataxia in the south of Italy: a clinical and biochemical survey of 23 patients. Can $\mathcal{f}$ Neurol Sci 1980;7:351-7.

26 DeFalco FA, Mansi D, Ventola F, Filla A, Campanella G. Proposta di una scheda di rilevamento clinico delle atassie spinocerebellari. Acta Neurol 1979;39:103-9.

27 Polinsky RJ, Brown RT, Burns RS, Harvey-White J, Kopin IJ. Low lumbar CSF concentrations of homovanillic acid and 5-hydroxyindoleacetic acid in multiple system atrophy with autonomic failure. $\mathcal{F}$ Neurol Neurosurg Psychiatry phy with autonomic

28 Wilcoxon $\mathrm{F}$. Individual comparisons by ranking methods. Biometrics 1945;1:80-3.

29 Snedecor GW, Cochran WG. The binomial distribution In: Statistical methods. 8th ed. Iowa: Ames Iowa State University Press, 1989:116-76.

30 Takiyama Y. A clinical and pathologic study of a large Japanese family with Machado-Joseph disease tightly linked to the DNA markers on chromosome 149 Neurology 1994;44:1302-8.

31 Robitaille Y, Schut L, Kish SJ. Structural and immunocytochemical features of olivopontocerebellar atrophy caused by spinocerebellar ataxia type 1 (SCA-1) mutation defines a unique phenotype. Acta Neuropathol 1995; 90:572-81.

32 Dürr A, Stevanin G, Cancel G, et al. Spinocerebellar ataxia 3 and Machado-Joseph disease: clinical molecular and 3 and Machado-Joseph disease: clinical molecular

33 Silviera I, Lopes-Cendes I, Kish S, et al. Frequency of spinocerebellar ataxia type 1, dentatorubropallidoluysian atrophy and Machado-Joseph disease mutations in a large group of spinocerebellar ataxia mutations. Neurology 1996;46:214-18. 